\title{
Extreme mass ratio binary: radiation reaction and gravitational waveform
}

\author{
Yasushi Mino \\ Mail Code 130-33, Caltech Pasadena, CA 91125, USA \\ E-mail: mino@tapir.caltech.edu
}

Received 19 October 2004, in final form 17 December 2004

Published 26 April 2005

Online at stacks.iop.org/CQG/22/S375

\begin{abstract}
For a successful detection of gravitational waves by LISA, it is essential to construct theoretical waveforms in a reliable manner. We discuss gravitational waves from an extreme mass ratio binary system which is expected to be a promising target of the LISA project. The extreme mass ratio binary is a binary system of a supermassive black hole and a stellar mass compact object. As the supermassive black hole dominates the gravitational field of the system, we suppose that the system might be well approximated by a metric perturbation of a Kerr black hole. We discuss recent theoretical progress in calculating the waveforms from such a system.
\end{abstract}

PACS numbers: $04.25 . \mathrm{Nx}, 04.30 .-\mathrm{w}$

\section{Introduction}

We consider a method to construct theoretical templates of gravitational waves LISA will detect. We are planning to operate LISA around a few years, and we may observe gravitational waves for this time scale. One promising target for LISA is a binary system of a supermassive black hole and an inspiralling compact object, and we discuss a method to calculate a gravitational waveform coming from such a system. Since the supermassive black hole dominates the gravitational field of the system, we expect that a linear metric perturbation of a Kerr black hole induced by a point particle might be a useful tool for this calculation.

Because we already have a method to calculate a metric perturbation of a given orbit of the particle, it was widely believed that the key issue here is to calculate a self-force, so that one can derive the orbit of the particle. We derived a formal expression of the self-force [1] and we come to have a promising method to calculate the self-force on the particle [2]. However, there was a concern about this approach because of a gauge dependence of the self-force.

We discuss that the self-force is totally gauge dependent. By a special choice of the gauge condition, the self-force vanishes for the entire time interval where the linear metric 
perturbation is valid (see section 2). On the other hand, gravitational radiation reaction is observable by asymptotic gravitational waves, and we expect that a physically reasonable self-force must describe this effect. Thus, the vanishing self-force suggests that we need a substantial extension of the theoretical basis in calculating the self-force, especially, of the gauge condition.

We find a key idea to solve this problem is an adiabatic approximation of the metric perturbation, which is different from a usual metric perturbation scheme. The idea of an adiabatic approximation of the metric changes our understanding of the orbital evolution and the validity of the self-force was clarified for the first time. We find that the class of gauge conditions used in the radiation reaction formalism in [3] is compatible with the adiabatic approximation of the metric perturbation. With a further choice of the gauge condition, we find that the radiation reaction formula might describe the orbit sufficiently long enough for the LISA project.

\section{Self-force? A myth?}

Gravitational radiation reaction is physically real since we can define the gravitational flux at the asymptotically flat region, and we expect to see this effect on the orbit by a modulation of an observed gravitational waveform. In order to predict this modulation, we were motivated to calculate the self-force in the belief that the self-force tells us the gravitational radiation reaction effect on the orbit. However, we find that the self-force could vanish consistently with a usual perturbation method irrespective of gravitational radiation reaction.

For an approximation of an extreme mass ratio binary system, we take the mass ratio of the binary $\mu$ as a small parameter for the expansion. In a usual perturbation scheme, we expand the metric and the orbit from background quantities of the supermassive black hole as

$$
\begin{aligned}
& g_{\alpha \beta}=g_{\alpha \beta}^{\mathrm{kerr}}+\mu h_{\alpha \beta}^{(1)}+\mu^{2} h_{\alpha \beta}^{(2)}+\cdots, \\
& z^{\alpha}(\lambda)=z^{(\mathrm{kerr}) \alpha}(\lambda)+\mu z^{(1) \alpha}(\lambda)+\mu^{2} z^{(2) \alpha}(\lambda)+\cdots,
\end{aligned}
$$

where $g_{\alpha \beta}^{\text {kerr }}$ is a Kerr metric and $z^{(\mathrm{kerr}) \alpha}(\lambda)$ is a geodesic around it with $\lambda$ as an orbital parameter. For a valid perturbation, we assume

$$
\begin{aligned}
& O(1) \sim g_{\alpha \beta}^{b g}>\mu h_{\alpha \beta}^{(1)}>\mu^{2} h_{\alpha \beta}^{(2)}>\cdots \\
& O(1) \sim z^{(b g) \alpha}(\lambda)>\mu z^{(1) \alpha}(\lambda)>\mu^{2} z^{(2) \alpha}(\lambda)>\cdots
\end{aligned}
$$

and the perturbative equations are derived by expanding the Einstein equation with respect to $\mu$.

One can see that this perturbation scheme allows only a small deviation of the orbit from the background geodesic. Because the orbit will deviate from the background geodesic eventually by gravitational radiation reaction, this perturbation scheme is valid in a finite time interval.

We consider a gauge transformation of the orbit in this finite time interval where the perturbation is valid. Because the deviation from a geodesic is $O(\mu)$, one can always take a gauge transformation to eliminate the deviation during this whole time interval, i.e. $\mu z^{(1) \alpha}=\mu^{2} z^{(2) \alpha}=0$, thus, the self-force totally vanishes consistently with the usual perturbation scheme. 


\section{Adiabatic metric perturbation}

The reason for this gauge problem of the self-force is that the perturbation is valid only in a short time scale, so that we can only describe a small deviation of the orbit from the background geodesic. In order to solve this problem, we propose an extension of the perturbation scheme.

Bound geodesics around a Kerr black hole with a convenient orbital parameter $\lambda$ are characterized by three constants of motion $\mathcal{E}^{a}, a=E, L, C$, and four phase constants $\lambda^{b}, b=r, \theta, C^{c}, c=t, \phi$ as in [3], and we denote them collectively by $\gamma=\left\{\mathcal{E}^{a}, \lambda^{b}, C^{c}\right\}$. From the result of [3], the orbital deviation becomes $O(1)$ when $\lambda \sim O\left(\mu^{-1 / 2}\right)$. This time scale is called the dephasing time. From the discussion in section 2 , the usual perturbation scheme is valid only in this time scale. Our purpose here is to extend this time scale of validity by modifying the metric perturbation scheme.

In the usual perturbation scheme, the linear metric perturbation is induced by a geodesic, and we can write it as a function of $\gamma, h_{\alpha \beta}(x)=h_{\alpha \beta}(\gamma ; x)$. Here we use a class of gauge conditions where the metric perturbation is derived by the Green function in the Boyer-Lindquist coordinates as

$$
\begin{aligned}
& h_{\alpha \beta}(x)=\int \mathrm{d} x^{\prime} G_{\alpha \beta \alpha^{\prime} \beta}\left(x, x^{\prime}\right) T^{\alpha^{\prime} \beta^{\prime}}\left(x^{\prime}\right), \\
& G_{\alpha \beta \alpha^{\prime} \beta^{\prime}}\left(x, x^{\prime}\right)=G_{\alpha \beta \alpha^{\prime} \beta^{\prime}}\left(t-t^{\prime}, \phi-\phi^{\prime}, r, r^{\prime}, \theta, \theta^{\prime}\right) .
\end{aligned}
$$

We assume an adiabatic approximation to the orbit as in [3], and consider the evolution of 'geodesic constants' by the effect of gravitational radiation reaction. We write the 'geodesic constants' as functions of the orbital parameter, $\gamma(\lambda)$. We consider extending this idea to the metric perturbation. We foliate the spacetime by spacelike hypersurfaces. Using the fact that the foliation surfaces intersect with the orbit, we define the foliation function $f(x)$ by the orbital parameter at the intersection of the orbit and the surfaces as $f(z(\lambda))=\lambda$. We define the adiabatic metric perturbation on the foliation surface $f(x)$ by the linear metric perturbation induced by the geodesic $\gamma(f)$ as

$$
h_{\alpha \beta}^{a d}(x)=h_{\alpha \beta}(\gamma(f) ; x) .
$$

In order to see the validity of the adiabatic metric perturbation, we operate the linearized Einstein operator and we have

$$
G_{\alpha \beta}^{(1)}\left[h^{a d}\right]=T_{\alpha \beta}[\gamma(f)]+\Lambda_{\alpha \beta}^{(1)}\left[h^{a d}\right]
$$

where an extra term $\Lambda_{\alpha \beta}^{(1)}$ appears because the adiabatic metric perturbation is not induced by a geodesic. It is notable that $T_{\alpha \beta}[\gamma(f)]$ is the stress-energy tensor of a point particle moving along the orbit with the effect of gravitational radiation reaction. The adiabatic metric perturbation solves the Einstein equation to the accuracy of $O(\mu)$ as long as $O(\mu)>\Lambda_{\alpha \beta}^{(1)}$ holds. Since $\Lambda_{\alpha \beta}^{(1)}$ depends on the $\lambda$ derivatives of the orbital 'constants' $\gamma(\lambda)$, the validity of the adiabatic metric perturbation depends on how the orbit evolves. We find that, under the gauge condition (3.2), the adiabatic metric perturbation is valid in the radiation reaction time scale $O\left(\mu^{-1}\right)>\lambda$.

Because the time scale of validity is much longer than that of the linear perturbation, the orbital deviation could be $O(1)$ in this metric perturbation scheme and one cannot eliminate the orbital deviation from the geodesic by a gauge transformation, thus, the self-force cannot vanish by a gauge transformation and is physically meaningful to predict the orbit in this class of gauge conditions. 


\section{Adiabatic evolution of the orbit}

Reference [3] finds that the infinite time averaged self-force acting on the 'constants of motion', $\left\langle(\mathrm{d} / \mathrm{d} \lambda) \mathcal{E}^{a}\right\rangle$, can be derived by the radiative metric perturbation in a gauge invariant way by a simple symmetry property of a Kerr spacetime. It also finds that $\left\langle(\mathrm{d} / \mathrm{d} \lambda) \mathcal{E}^{a}\right\rangle$ makes a dominant contribution to the orbital evolution by a perturbative analysis of the orbit.

The adiabatic metric perturbation is valid in the radiation reaction time scale and, in this time scale, the orbital evolution is non-perturbative. As for the 'constants of motion', one can still deal with the evolution by a perturbation, and we find that $\left\langle(\mathrm{d} / \mathrm{d} \lambda) \mathcal{E}^{a}\right\rangle$ dominantly determines the evolution of them. The subdominant part of the 'constants of motion' is $O(\mu)$. The evolution of the 'phase constants' becomes non-perturbative beyond the dephasing time; however, we find the perturbative results in [3] are qualitatively correct. The dominant part of their evolution is described only by $\left\langle(\mathrm{d} / \mathrm{d} \lambda) \mathcal{E}^{a}\right\rangle$, and the subdominant part behaves as $O(\mu \lambda)$.

Because the subdominant part of the evolution of 'constants of motion' is smaller than $O(1)$ in the radiation reaction time scale, one can find a gauge condition which can totally eliminate this part. We call this gauge condition the radiation reaction gauge, and, in this gauge, the self-force can be written only by the infinite time averaged self-force as

$$
(\mathrm{d} / \mathrm{d} \lambda) \mathcal{E}^{a}=\left\langle(\mathrm{d} / \mathrm{d} \lambda) \mathcal{E}^{a}\right\rangle .
$$

The self-force is expected to have a conservative part as well as a radiative part, however, in this gauge condition, the conservative part is integrated out and appears to be the small shift of initial values. We also note that the radiation reaction gauge condition applies to an orbit of a spinning particle. It is known that the orbit of the spinning test particle slightly deviates from a geodesic [5] because of the coupling of its spin and the background curvature. This effect of the deviation can also be absorbed by a small change of the initial values by the radiation reaction gauge condition.

\section{Self-force calculation versus radiation reaction formula}

Here we try to compare these two approaches to calculate the orbit; the regularization calculation of the self-force [2] and the radiation reaction formula discussed in section 4 . There could be two issues for comparison;

(1) Theoretical issue; whether the resulting orbit is physically acceptable or not

(2) Practical issue; whether the approach is practically available for generating templates for the LISA project.

Gravitational radiation reaction is a physically real effect. Because the momentum flux carried by gravitational waves is well defined, it is reasonable to expect that the orbital evolution must be consistent to this effect. There is a widespread belief that the self-force includes the effect of gravitational radiation reaction; however, this is not trivial in the usual perturbation scheme because of the gauge freedom as we argue in section 2 . We consider that the regularization calculation of the self-force [2] is not guaranteed to include this radiation reaction effect in general. On the other hand, the radiation reaction formula uses a certain class of gauge conditions as we argue in section 3 , and the orbital evolution is described consistently with asymptotic gravitational radiation.

For a practical issue, an idea is proposed to calculate the metric perturbation in a Kerr black hole available for the self-force calculation [6]; however, it is not coded yet, and a more crucial problem is whether the resulting self-force is consistent with gravitational radiation reaction. Even if it is possible, the numerical convergence of the self-force is not optimal since 
it is a point splitting regularization. On the other hand, the radiation reaction formula is, in the end, just an extension of the balance formula, and various numerical codes of the balance formula were already made successfully [7]. There is also known a semi-analytic technique which may substantially increase the efficiency of the code [8]. In this approach, we have the most optimal convergence because the regularization is done in a quite natural manner, and we consider it helpful to have an efficient calculation.

\section{Acknowledgments}

YM thanks Professor Kip Thorne and Professor Sterl Phinney for encouragement. YM was supported by NASA grant NAG5-12834 and NASA-ATP grant NNG04GK98G at CalTech.

\section{References}

[1] Some review papers on this issue are; Poisson E 2004 Living Rev. Rel. 6 Mino Y, Sasaki M and Tanaka T 1997 Prog. Theor. Phys. Suppl. 128373

[2] Detweiler S, Messaritaki E and Whiting B F 2003 Phys. Rev. D 67104016 Barack L and Ori A 2003 Phys. Rev. Lett. 90111101

Barack L and Ori A 2003 Phys. Rev. D 67024029

Mino Y, Nakano H and Sasaki M 2003 Prog. Theor. Phys. 1081039

Barack L and Lousto C O 2002 Phys. Rev. D 66061502

Barack L and Ori A 2002 Phys. Rev. D 66084022

Barack L, Mino Y, Nakano H, Ori A and Sasaki M 2002 Phys. Rev. Lett. 88091101

[3] Mino Y 2003 Phys. Rev. D 67084027

[4] Mino Y In preparation

[5] Papapetrou A 1951 Proc. R. Soc. Lon. A 209243

[6] Ori A 2003 Phys. Rev. D 67124010

[7] Recent numerical calculations of high precision are:

Glampedakis K and Kennefick D 2002 Phys. Rev. D 66044002

Hughes S A 2001 Phys. Rev. D 64064004

Hughes S A 2000 Phys. Rev. D 61084004

Hughes S A 2001 Phys. Rev. D 63049902 (erratum)

[8] Fujita R and Tagoshi H 2004 Prog. Theor. Phys. 112415

Mano S and Takasugi E 1997 Prog. Theor. Phys. 97213

Mano S, Suzuki H and Takasugi E 1996 Prog. Theor. Phys. 951079 